\title{
Finite-temperature Casimir effect in the presence of nonlinear dielectrics
}

\author{
Kheirandish, Fardin; Amooghorban, Ehsan; Soltani, Morteza
}

Published in:

Physical Review A

Link to article, DOI:

10.1103/PhysRevA.83.032507

Publication date:

2011

Document Version

Publisher's PDF, also known as Version of record

Link back to DTU Orbit

Citation (APA):

Kheirandish, F., Amooghorban, E., \& Soltani, M. (2011). Finite-temperature Casimir effect in the presence of nonlinear dielectrics. Physical Review A, 83(3), 032507. https://doi.org/10.1103/PhysRevA.83.032507

\section{General rights}

Copyright and moral rights for the publications made accessible in the public portal are retained by the authors and/or other copyright owners and it is a condition of accessing publications that users recognise and abide by the legal requirements associated with these rights.

- Users may download and print one copy of any publication from the public portal for the purpose of private study or research.

- You may not further distribute the material or use it for any profit-making activity or commercial gain

- You may freely distribute the URL identifying the publication in the public portal 


\title{
Finite-temperature Casimir effect in the presence of nonlinear dielectrics
}

\author{
Fardin Kheirandish, ${ }^{1, *}$ Ehsan Amooghorban, ${ }^{1,2, \dagger}$ and Morteza Soltani ${ }^{1, \ddagger}$ \\ ${ }^{1}$ Department of Physics, Faculty of Science, University of Isfahan, Isfahan, Iran \\ ${ }^{2}$ Department of Photonics Engineering, Technical University of Denmark, DK-2800 Kgs. Lyngby, Denmark \\ ${ }^{3}$ Department of Physics, Faculty of Science, University of Isfahan, Isfahan, Iran
}

(Received 3 February 2011; published 23 March 2011)

\begin{abstract}
Starting from a Lagrangian, the electromagnetic field in the presence of a nonlinear dielectric medium is quantized using path-integral techniques, and correlation functions of different fields are calculated. The susceptibilities of the nonlinear medium are obtained, and their relations to coupling functions are determined. Finally, the Casimir energy and force in the presence of a nonlinear medium at finite temperature are calculated.
\end{abstract}

\section{INTRODUCTION}

One of the most direct manifestations of the zero-point vacuum oscillations is the Casimir effect. This effect in its simplest form is the attractive force between two neutral, infinitely large, parallel, and ideal conductors in a vacuum. The effect is completely quantum mechanical and is a result of electromagnetic-field quantization in the presence of some boundary conditions. The presence or absence of boundary conditions causes a finite change in the vacuum energy whose variation with respect to the distance between the conductors creates the Casimir force [1]. The general theory of forces between parallel dielectrics was worked out by Lifshitz et al. [2]. Some years later, the theory of the Casimir effect was rederived by Schwinger et al. [3]. Later, this theory was extended to systems consisting of some dielectric layers [4,5], and precision experiments on measuring the Casimir force were performed [6-9].

Generally, calculations of Casimir forces are based on two different approaches [10]. In the first approach, the total energy of discrete quantum modes of the electromagnetic field is calculated. Usually, a regularization procedure is required, although there is still no consensus on them, which often lead to inconsistent results [11]. On the other hand, this formalism, despite its simplicity, is restricted to systems where energy eigenvalues are known, but geometries in which energy eigenvalues are known exactly are few.

The second approach is based on a Green's-function method, where using the fluctuation-dissipation theorem, the electromagnetic-field energy density is linked directly to the photonic Green's function.

In recent years, considerable attention has been paid to the Casimir effect because of its wide applications in different areas of physics, such as quantum field theory, gravitation and cosmology, nanotechnology, and atomic, condensed-matter, and mathematical physics [12-15]. On the other hand, during the last two decades, the emergence of periodically structured optical materials, commonly called photonic crystals, has led to substantial progress in the science and technology of optics and photonics [16]. Photonic crystals [17,18] provide

\footnotetext{
*fardin_kh@phys.ui.ac.ir

†amooghorban@sci.ui.ac.ir

${ }^{\ddagger}$ msoltani@phys.ui.ac.ir
}

new opportunities for enhancing and controlling nonlinear optical processes. An important step in this direction would be to provide a quantum theory of light that takes into account nonlinear processes and at the same time includes the absorption in nonlinear material. Several attempts have been made to provide a quantized theory of macroscopic nonlinear electrodynamics [19-27], where most of them have focused on strictly lossless materials. In addition, ambiguities persist because of some important restrictive approximations. Recently, a consistent approach that includes absorption and dispersion has been formulated within the framework of quantum Brownian motion [28]. This motivated us to investigate the quantization of the electromagnetic field in the presence of a nonlinear dielectric medium and also consider the nonlinear effects of the medium on the Casimir effect which can have applications in both fundamental science and engineering.

In [29], Li and Kardar developed a path-integral approach for computing fluctuation-induced forces between manifolds immersed in a correlated fluid. Golestanian and Kardar extended this formalism to arbitrary but small dynamic deformations of the boundaries and focused on the mechanical response in a vacuum [30]. Emig et al. used the path-integral formalism to obtain the normal and lateral Casimir forces between two sinusoidal corrugated perfect conductor surfaces [31,32]. Recently, this formalism has been merged with a canonical approach to calculate the Casimir force between two perfectly conducting plates immersed in a magnetodielectric medium [33]. In this paper, the electromagnetic field in the presence of a nonlinear magnetodielectric medium is quantized in the framework of path integrals, and, as an example, the mutual Casimir force between conducting parallel plates that are immersed in a nonlinear dielectric medium is calculated.

The layout of this paper is as follows: In Sec. II, a Lagrangian for the electromagnetic field in the presence of a nonlinear medium is proposed, and quantization is achieved via path integrals. In Secs. III and IV, the linear and nonlinear Green's functions of the electromagnetic field and medium are obtained, and the $n$ th-order susceptibility of the nonlinear medium that satisfies the Kramers-Kronig relations is determined. In Sec. V, the Casimir forces in the presence of linear and nonlinear media for zero and finite temperatures are calculated. Finally, we discuss the main results and conclude in Sec. VI. 


\section{FIELD QUANTIZATION}

Quantum electrodynamics in a linear magnetodielectric medium can be accomplished by modeling the medium with two independent reservoirs interacting with the electromagnetic field. Each reservoir contains a continuum of three-dimensional harmonic oscillators describing the electric and magnetic properties of the medium [34-36]. In this section, we follow the idea introduced in [28] to quantize the electromagnetic field in the presence of a nonlinear medium, and for simplicity, we restrict our attention to a nonmagnetic medium, but the generalization to a magnetodielectric medium is straightforward. For this purpose, let us consider the following total Lagrangian density:

$$
\mathcal{L}=\mathcal{L}_{\text {EM }}+\mathcal{L}_{\text {mat }}+\mathcal{L}_{\text {int }},
$$

where $\mathcal{L}_{\mathrm{EM}}$ is the electromagnetic-field Lagrangian density

$$
\mathcal{L}_{\mathrm{EM}}=\frac{1}{2} \mathbf{E}^{2}-\frac{1}{2} \mathbf{B}^{2}
$$

The physical fields can be written in terms of the potentials as $\mathbf{E}=-\frac{\partial \mathbf{A}}{\partial t}-\nabla \phi$ and $\mathbf{B}=\nabla \times \mathrm{B}$, and for simplicity we work in the axial gauge where $\phi=0$. The dielectric medium is modeled by a vector field $\mathbf{X}(\omega)$, which is used to describe its electrical properties

$$
\mathcal{L}_{\text {mat }}=\int_{0}^{\infty} d \omega \frac{1}{2} \dot{\mathbf{X}}^{2}(\omega, x)-\frac{1}{2} \omega^{2} \mathbf{X}^{2}(\omega, x) .
$$

Now, we define the $i$ th component of the polarization field of the dielectric medium as

$$
\begin{aligned}
P_{i}(x)= & \int_{0}^{\infty} d \omega v^{(1)}(\omega) X_{i}(\omega, x)+\int_{0}^{\infty} d \omega \int_{0}^{\infty} d \omega^{\prime} v^{(2)}\left(\omega, \omega^{\prime}\right) X_{i}(\omega, x) X_{i}\left(\omega^{\prime}, x\right) \\
& +\int_{0}^{\infty} d \omega \int_{0}^{\infty} d \omega^{\prime} \int_{0}^{\infty} d \omega^{\prime \prime} v^{(3)}\left(\omega, \omega^{\prime}, \omega^{\prime \prime}\right) X_{i}(\omega, x) X_{i}\left(\omega^{\prime}, x\right) X_{i}\left(\omega^{\prime \prime}, x\right)+\cdots
\end{aligned}
$$

In this expression, the index $i$ can take on the values $x$, $y$, and $z$, and $v^{(1)}, v^{(2)}, v^{(3)}, \ldots$ are the coupling functions between the medium and the electromagnetic field. As can be seen from Eq. (4), the coupling tensor $v^{(1)}$ describes the linear contribution of the interaction, and the sequence $v^{(2)}, v^{(3)}, \ldots$ describes, respectively, the first-order, secondorder, and higher-order nonlinear interactions. The coupling tensors $v^{(1)}, v^{(2)}, v^{(3)}, \ldots$ in Eq. (4) are the key parameters in this quantization scheme, and we will see that the susceptibility functions of the medium can be expressed in terms of these coupling tensors.

The interaction part of Lagrangian is defined by [28]

$$
\mathcal{L}_{\text {int }}=\mathbf{A} \cdot \dot{\mathbf{P}} .
$$

Now, we quantize the theory using the path-integral techniques. For this purpose, let us start with the following generating functional [37]:

$$
Z[J]=\int \mathcal{D}[\varphi] \exp i \int d^{4} x[\mathcal{L}(\varphi(x))+J(x) \varphi(x)]
$$

where $\varphi$ is a scalar field, $J$ acts as a source or an auxiliary field, and different correlation functions can be found by taking repeated functional derivatives with respect to the field $J(x)$.
The above partition function is Gaussian since the integrand is quadratic in fields. To obtain the generating functional for the interacting fields, we first calculate the generating functional for the free fields

$$
\begin{aligned}
Z_{0}\left[\mathbf{J}_{\mathrm{EM}},\left\{\mathbf{J}_{\omega}\right\}\right]= & \int \mathcal{D}[\mathbf{A}] \mathcal{D}[\mathbf{X}] \\
& \times \exp \left[i \int d ^ { 4 } x \left\{\mathcal{L}_{\mathrm{EM}}+\mathcal{L}_{\mathrm{mat}}+\mathbf{J}_{\mathrm{EM}} \cdot \mathbf{A}\right.\right. \\
& \left.\left.+\int d \omega \mathbf{J}_{\omega} \cdot \mathbf{X}\right\}\right]
\end{aligned}
$$

Using the four-dimensional version of Gauss's theorem,

$$
\int d^{4} x \mathcal{L}_{\mathrm{EM}}=-\int d^{4} x\left[\mathbf{A} \cdot(\nabla \times \nabla \times \mathbf{A})+\mathbf{A} \cdot \partial_{t}^{2} \mathbf{A}\right],
$$

and using integration by parts,

$$
\begin{aligned}
& \int d^{4} x \dot{\mathbf{X}}(\omega, x) \cdot \dot{\mathbf{X}}(\omega, x) \\
& =-\int d^{4} x \mathbf{X}(\omega, x) \cdot \frac{\partial^{2}}{\partial t^{2}} \mathbf{X}(\omega, x) .
\end{aligned}
$$

From Eqs. (8) and (9), the free-field generating functional [Eq. (7)] can be written as

$$
\begin{aligned}
Z_{0}\left[\mathbf{J}_{\mathrm{EM}}, \mathbf{J}_{\omega}\right]= & \int \mathcal{D}[\mathbf{A}] \mathcal{D}[\mathbf{X}] \exp -\frac{i}{2}\left[\int d ^ { 4 } x \left\{\left(\mathbf{A} \cdot \nabla \times \nabla \times \mathbf{A}+\mathbf{A} \cdot \partial_{t}^{2} \mathbf{A}\right)-\mathbf{J}_{\mathrm{EM}} \cdot \mathbf{A}\right.\right. \\
& \left.\left.+\int d \omega \mathbf{X}(\omega, x)\left(\frac{\partial^{2}}{\partial t^{2}}+\omega^{2}\right) \mathbf{X}(x, \omega)-\mathbf{J}_{\omega}(x) \cdot \mathbf{X}(x, \omega)\right\}\right] .
\end{aligned}
$$

The integral in Eq. (10) can be easily calculated from the field version of the quadratic integral formula, and the result is

$$
Z_{0}\left[\mathbf{J}_{\mathrm{EM}}, \mathbf{J}(\omega)\right]=\exp \frac{i}{2}\left[\int d^{4} x \int d^{4} x^{\prime} \mathbf{J}_{\mathrm{EM}} \cdot \stackrel{\leftrightarrow}{G}_{\mathrm{EM}}^{(0)}\left(x-x^{\prime}\right) \cdot \mathbf{J}_{\mathrm{EM}}+\int d^{3} \mathbf{x} \int d t \int d t^{\prime} \int d \omega \mathbf{J}_{\omega}(\mathbf{x}, t) \cdot \stackrel{\leftrightarrow}{G}_{\omega}\left(t-t^{\prime}\right) \cdot \mathbf{J}_{\omega}\left(\mathbf{x}, t^{\prime}\right)\right],
$$


where the space component of the point $x \in \mathbb{R}^{4}$ is indicated in bold by $\mathbf{x} \in \mathbb{R}^{3}$, and the time component is indicated by $t$ or $x_{0} \in \mathbb{R}$. The Green's functions $\stackrel{\leftrightarrow}{G}_{\mathrm{EM}}^{(0)}\left(x-x^{\prime}\right)$ and $\stackrel{\leftrightarrow}{G}_{\omega}\left(t-t^{\prime}\right)$ are the propagators for free fields and satisfy the following equations:

$$
\begin{gathered}
\left(\nabla \times \nabla \times+\frac{\partial^{2}}{\partial t^{2}}\right) \stackrel{\leftrightarrow}{G}_{\mathrm{EM}}^{(0)}\left(x-x^{\prime}\right)=\delta\left(x-x^{\prime}\right), \\
\left\{\frac{\partial^{2}}{\partial t^{2}}+\omega^{2}\right\} \stackrel{\leftrightarrow}{G}_{\omega \alpha, \beta}\left(t-t^{\prime}\right)=\delta\left(t-t^{\prime}\right) \delta_{\alpha, \beta} .
\end{gathered}
$$

Taking the Fourier transform of Eqs. (12) and (13), we find

$$
\begin{gathered}
\stackrel{\leftrightarrow}{G}_{\mathrm{EM}, \alpha \beta}^{(0) \|}(\mathbf{k}, \omega)=-\frac{k_{\alpha} k_{\beta}}{\omega^{2}}, \\
\stackrel{\leftrightarrow}{G}_{\mathrm{EM}, \alpha \beta}^{(0) \perp}(\mathbf{k}, \omega)=\frac{\delta_{\alpha \beta}-\widetilde{k}_{\alpha} \widetilde{k}_{\beta}}{\mathbf{k}^{2}-\omega^{2}},
\end{gathered}
$$

and

$$
G_{\omega \alpha \beta}\left(\omega^{\prime}\right)=\frac{\delta_{\alpha \beta}}{\omega^{2}-\left(\omega^{\prime}+i 0^{+}\right)^{2}},
$$

where $\widetilde{\mathbf{k}}$ is a unit vector along $\mathbf{k}$, and $\stackrel{\leftrightarrow}{G}^{\|}$and $\stackrel{\leftrightarrow}{G}^{\perp}$ refer to the longitudinal and transverse parts of the Green's tensor in Fourier space. For further simplicity, we define

$$
\begin{aligned}
J_{i, P}= & \int d \omega v^{(1)}(\omega) J_{i, \omega}+\int d \omega \int d \omega / \nu^{(2)}\left(\omega, \omega^{\prime}\right) J_{i, \omega} J_{i, \omega^{\prime}} \\
& +\int d \omega \int d \omega^{\prime} \nu^{(3)}\left(\omega, \omega^{\prime}, \omega^{\prime \prime}\right) J_{i, \omega} J_{i, \omega^{\prime}} J_{i, \omega^{\prime \prime}}+\cdots
\end{aligned}
$$

Now, the generating functional of the interacting fields can be written in terms of the free generating functional as [38]

$$
\begin{aligned}
Z\left[\mathbf{J}_{0}, \mathbf{J}_{P}\right]= & Z^{-1}[0] \exp \left[i \int d^{4} z \mathcal{L}_{\mathrm{int}}\left(\frac{1}{i} \frac{\delta}{\delta \mathbf{J}_{\mathrm{EM}}(z)}, \frac{1}{i} \frac{\delta}{\delta \mathbf{J}_{P(z)}}\right)\right] Z_{0}\left[\mathbf{J}_{\mathrm{EM}}, \mathbf{J}_{\omega}\right] \\
= & Z^{-1}[0] \sum_{n=0}^{\infty} \frac{1}{n !}\left\{i \int d ^ { 4 } z \left[\int d \omega_{1} v^{(1)}\left(\omega_{1}\right) \frac{1}{i} \frac{\delta}{\delta \mathbf{J}_{\mathrm{EM}}(z)} \frac{\partial}{\partial z_{0}} \frac{1}{i} \frac{\delta}{\delta \mathbf{J}_{\omega_{1}}(z)}\right.\right. \\
& +\int d \omega_{1} \int d \omega_{2} v^{(2)}\left(\omega_{1}, \omega_{2}\right) \frac{1}{i} \frac{\delta}{\delta \mathbf{J}_{\mathrm{EM}}(z)} \frac{\partial}{\partial z_{0}} \frac{1}{i} \frac{\delta}{\delta \mathbf{J}_{\omega_{1}}(z)} \frac{1}{i} \frac{\delta}{\delta \mathbf{J}_{\omega_{2}}(z)} \\
& \left.\left.+\int d \omega_{1} \int d \omega_{2} \int d \omega_{3} v^{(3)}\left(\omega_{1}, \omega_{2}, \omega_{3}\right) \frac{1}{i} \frac{\delta}{\delta \mathbf{J}_{\mathrm{EM}}(z)} \frac{\partial}{\partial z_{0}} \frac{1}{i} \frac{\delta}{\delta \mathbf{J}_{\omega_{1}}(z)} \frac{1}{i} \frac{\delta}{\delta \mathbf{J}_{\omega_{2}}(z,)} \frac{1}{i} \frac{\delta}{\delta \mathbf{J}_{\omega_{3}}(z)}+\cdots\right]\right\}^{n} Z_{0}\left[\mathbf{J}_{\mathrm{EM}}, \mathbf{J}_{\omega}\right]
\end{aligned}
$$

where $Z[0]$ is a normalization factor.

\section{LINEAR GREEN'S TENSORS}

In this section, we consider a linear medium, so the nonlinear coupling functions $v^{(2)}, v^{(3)}$, etc., are zero. Following the procedure presented in [33], the linear Green's function of the electromagnetic field can be obtained as

$$
\stackrel{\leftrightarrow}{G}_{\alpha \beta}^{(1)}(x-y)=-\left.i \frac{\delta^{2} Z[J]}{\delta J_{\alpha}(x) \delta J_{\beta}(y)}\right|_{J=0} .
$$

To facilitate the calculations, we take the time-Fourier transform of the Green's function (19). After some lengthy but straightforward calculations, we find the Green's function in frequency space as

$$
\begin{aligned}
& \stackrel{\leftrightarrow}{G}_{\mathrm{EM}}^{(1)}\left(\mathbf{x}-\mathbf{x}^{\prime}, \omega\right)=\stackrel{\leftrightarrow}{G}_{0}\left(\mathbf{x}-\mathbf{x}^{\prime}, \omega\right)+\int d \mathbf{x}_{1}\left[\stackrel{\leftrightarrow}{G}_{0}\left(\mathbf{x}-\mathbf{x}_{1}, \omega\right)\right. \\
& \left.\times \int d \omega^{\prime}\left\{v^{2}\left(\omega^{\prime}\right) \omega^{2} \stackrel{\leftrightarrow}{G}_{\omega^{\prime}}(\omega)\right\} \stackrel{\leftrightarrow}{G}_{\mathrm{EM}}^{(1)}\left(\mathbf{x}_{1}-\mathbf{x}^{\prime}, \omega\right)\right]
\end{aligned}
$$

It can be shown that this Green's function satisfies the following equation:

$$
\nabla \times \nabla \times \stackrel{\leftrightarrow}{G}_{\mathrm{EM}}^{(1)}\left(\mathbf{x}-\mathbf{x}^{\prime}, \omega\right)-\omega^{2} \epsilon(\omega) \stackrel{\leftrightarrow}{G}_{\mathrm{EM}}^{(1)}\left(\mathbf{x}-\mathbf{x}^{\prime}, \omega\right)
$$

$$
=\overleftrightarrow{\delta}\left(\mathbf{x}-\mathbf{x}^{\prime}\right)
$$

with the formal solution

$$
\begin{gathered}
\stackrel{\leftrightarrow}{G}_{\mathrm{EM}, \alpha \beta}^{(1) \|}(\mathbf{k}, \omega)=-\frac{k_{\alpha} k_{\beta}}{\omega^{2} \epsilon(\omega)}, \\
\stackrel{\leftrightarrow}{G}_{\mathrm{EM}, \alpha \beta}^{(1) \perp}(\mathbf{k}, \omega)=\frac{\delta_{\alpha \beta}-\widetilde{k}_{\alpha} \widetilde{k}_{\beta}}{\mathbf{k}^{2}-\omega^{2} \epsilon(\omega)},
\end{gathered}
$$

where $\epsilon(\omega)=1+\chi^{(1)}(\omega)$ is the linear permittivity of the medium in the frequency domain and the linear susceptibility $\chi^{(1)}(\omega)$ can be written as

$$
\chi^{(1)}(\omega)=\int_{0}^{\infty} d \omega^{\prime} \frac{\left[\nu^{(1)}\left(\omega^{\prime}\right)\right]^{2}}{\omega^{\prime 2}-\left(\omega+i 0^{+}\right)^{2}} .
$$

These are complex functions of frequency which satisfy Kramers-Kronig relations and have the properties of response functions, i.e., $\epsilon\left(-w^{*}\right)=\epsilon^{*}(\omega)$. It can be shown that these functions have no poles in the upper half-plane and tend to unity as $\omega \rightarrow \infty$. As a consequence, the electric susceptibility of the medium in the time domain can be written as

$$
\chi^{(1)}(t)=\int_{0}^{\infty} d \omega \frac{\sin \omega t}{\omega}\left[v^{(1)}(\omega)\right]^{2} \Theta(t),
$$

which is real and causal, and $\Theta(t)$ is the Heaviside step function. This proves that the constitutive relations of the 
medium, with arbitrary dispersion and absorption, are properly described by the present model. If we are given a definite permittivity for the medium, then we can invert the relations [Eq. (24)] and find the corresponding coupling function $v^{(1)}(\omega)$ as

$$
v^{(1)}(\omega)=\sqrt{\frac{2 \omega}{\pi} \operatorname{Im} \chi^{(1)}(\omega)} .
$$

In a similar way, we can obtain the other correlation functions among different fields [33], but here we consider only the correlation function

$$
\stackrel{\leftrightarrow}{G}_{\mathrm{EM}, P}^{(1)}\left(\mathbf{x}-\mathbf{x}^{\prime}, \omega\right)=i \omega \chi(1)(\omega) \stackrel{\leftrightarrow}{G}_{\mathrm{EM}}^{(1)}\left(\mathbf{x}-\mathbf{x}^{\prime}, \omega\right),
$$

which shows that $\chi^{(1)}(\omega)$ is a linear electric susceptibility [34,37]. In this way, we can easily define the nonlinear susceptibilities of the medium via two-, three-, and $n$-point correlation functions between the electromagnetic field and the polarization field [39]. These correlation functions satisfy the fluctuation-dissipation theorem $[32,39,40]$. In the next sections, we apply the path-integral formalism to calculate the Green's tensors and Casimir energy in the presence of a nonlinear dielectric medium. To simplify the problem, we work in Fourier space and separate the Green's tensor into transverse and longitudinal parts. The longitudinal part of the Green's function does not lead to any force since this part of the Green's function is local, and the local fields do not lead to any Casimir force [33]. Thus, we only consider the transverse part and, for simplicity, drop the superscript ${ }^{\perp}$.

\section{NONLINEAR GREEN'S TENSORS}

Now, let us consider the contributions induced from a nonlinear medium. To elucidate the method, we start with the term $v^{(2)}$ and generalize it to include the higher-order terms like $v^{(3)}, v^{(4)}$, etc. In analogy to the linear medium, we define the nonlinear Green's function of the electromagnetic field up to the first order of nonlinearity as

$$
\stackrel{\leftrightarrow}{G}_{\mathrm{EM}}^{(2)}(x-y)=-\left.i \frac{\delta^{2} Z[J]}{\delta J_{\mathrm{EM}}(x) \delta J_{\mathrm{EM}}(y)}\right|_{J=0} .
$$

The nonlinear Green's function (28), after some lengthy but straightforward calculations, can be obtained in reciprocal space as follows:

$$
\begin{aligned}
\stackrel{\leftrightarrow}{G}_{\mathrm{EM}}^{(2)}(\mathbf{k}, \omega) \approx & \stackrel{\leftrightarrow}{G}_{\mathrm{EM}}^{(1)}(\mathbf{k}, \omega)+\stackrel{\leftrightarrow}{G}_{\mathrm{EM}}^{(1)}(\mathbf{k}, \omega) \omega^{2} \Delta_{\mathrm{NL}}^{(2)}(\omega) \stackrel{G}{G}_{\mathrm{EM}}^{(1)}(\mathbf{k}, \omega) \\
& +\stackrel{\leftrightarrow}{G}_{\mathrm{EM}}^{(1)}(\mathbf{k}, \omega) \omega^{2} \Delta_{\mathrm{NL}}^{(2)}(\omega) \\
& \times \stackrel{\leftrightarrow}{G}_{\mathrm{EM}}^{(1)}(\mathbf{k}, \omega) \omega^{2} \Delta_{\mathrm{NL}}^{(2)}(\omega) \stackrel{\leftrightarrow}{G}_{\mathrm{EM}}^{(1)}(\mathbf{k}, \omega)+\cdots \\
& =\frac{1}{\mathbf{k}^{2}-\omega^{2}\left[\epsilon(\omega)+\Delta_{\mathrm{NL}}^{(2)}(\omega)\right]}
\end{aligned}
$$

where

$$
\begin{aligned}
\Delta_{\mathrm{NL}}^{(2)}(\omega)= & \frac{1}{2 \pi} \int_{0}^{\infty} d \omega_{1} \int_{0}^{\infty} d \omega_{2} \int_{-\infty}^{\infty} d \omega^{\prime}\left[v^{(2)}\left(\omega_{1}, \omega_{2}\right)\right]^{2} \\
& \times \frac{1}{\omega_{1}^{2}-\left(\omega-\omega^{\prime}+i 0^{+}\right)^{2}} \frac{1}{\omega_{2}^{2}-\left(\omega^{\prime}+i 0^{+}\right)^{2}}
\end{aligned}
$$

is the first-order correction to the Green's function of the electromagnetic field due to the nonlinearity of the medium. In fact, we have linearized Eq. (29) in terms of the first-order correction due to the nonlinearity of the medium, by only retaining the terms linear in $\Delta_{\mathrm{NL}}^{(2)}(\omega)$ and replacing $\stackrel{\leftrightarrow}{G}_{\mathrm{EM}}^{(0)}(\mathbf{k}, \omega)$ by $\stackrel{\leftrightarrow}{G}_{\mathrm{EM}}^{(1)}(\mathbf{k}, \omega)$ in Eq. (20). In a similar way, we find for $v^{(3)}$ that

$$
\stackrel{\leftrightarrow}{G}_{\mathrm{EM}}^{(3)}(\mathbf{k}, \omega)=\frac{1}{\mathbf{k}^{2}-\omega^{2}\left[\epsilon(\omega)+\Delta_{\mathrm{NL}}^{(2)}(\omega)+\Delta_{\mathrm{NL}}^{(3)}(\omega)\right]},
$$

where

$$
\begin{aligned}
\Delta_{\mathrm{NL}}^{(3)}(\omega)= & \frac{1}{(2 \pi)^{2}} \int_{0}^{\infty} d \omega_{1} \int_{0}^{\infty} d \omega_{2} \int_{0}^{\infty} d \omega_{3} \int_{-\infty}^{\infty} d \omega_{2}^{\prime} \int_{-\infty}^{\infty} d \omega_{3}^{\prime}\left[\nu^{(3)}\left(\omega_{1}, \omega_{2}, \omega_{3}\right)\right]^{2} \\
& \times \frac{1}{\omega_{1}^{2}-\left(\omega-\omega_{2}-\omega_{3}+i 0^{+}\right)^{2}} \frac{1}{\omega_{2}^{2}-\left(\omega_{2}^{\prime}+i 0^{+}\right)^{2}} \frac{1}{\omega_{3}^{2}-\left(\omega_{3}^{\prime}+i 0^{+}\right)^{2}} .
\end{aligned}
$$

Following this procedure, we find the nonlinear Green's function of the electromagnetic field up to the $n$th order of the nonlinearity as

$$
\stackrel{\leftrightarrow}{G}_{\mathrm{EM}}^{(n)}(\mathbf{k}, \omega)=\frac{1}{\mathbf{k}^{2}-\omega^{2}\left[\epsilon(\omega)+\Delta_{\mathrm{NL}}^{(2)}(\omega)+\Delta_{\mathrm{NL}}^{(3)}(\omega)+\cdots+\Delta_{\mathrm{NL}}^{(n)}(\omega)\right]},
$$

where

$$
\begin{aligned}
\Delta_{\mathrm{NL}}^{(n)}(\omega)= & \frac{1}{(2 \pi)^{n-1}} \int_{0}^{\infty} d \omega_{1} \int_{-\infty}^{\infty} d \omega_{2}^{\prime} \int_{0}^{\infty} d \omega_{2} \cdots \int_{-\infty}^{\infty} d \omega_{n}^{\prime} \int_{0}^{\infty} d \omega_{n}\left[v^{(n)}\left(\omega_{1}, \omega_{2}, \ldots, \omega_{n}\right)\right]^{2} \\
& \times \frac{1}{\omega_{1}^{2}-\left(\omega-\omega_{2}^{\prime}-\cdots-\omega_{n}^{\prime}+i 0^{+}\right)^{2}} \frac{1}{\omega_{2}^{2}-\left(\omega_{2}^{\prime}+i 0^{+}\right)^{2}} \cdots \frac{1}{\omega_{n}^{2}-\left(\omega_{n}^{\prime}+i 0^{+}\right)^{2}} .
\end{aligned}
$$

In a similar way, we can obtain correlation functions among different fields. For example, the three-points Green's function [41] up to the first order of nonlinearity is defined by

$$
\stackrel{\leftrightarrow}{G}_{\mathrm{EM}, \mathrm{EM}, P}^{(2)}\left(\mathbf{x}-\mathbf{x}^{\prime}, \mathbf{x}_{1}-\mathbf{x}^{\prime}, \omega_{1}, \omega_{2}\right)=-\left.\frac{\delta^{2} Z[J]}{\delta J_{\mathrm{EM}}(x) \delta J_{\mathrm{EM}}\left(x_{1}\right) \delta J_{\mathbf{P}}\left(x^{\prime}\right)}\right|_{J=0}=\omega_{1} \omega_{2} \chi^{(2)}\left(\omega_{1}, \omega_{2}\right) \stackrel{\leftrightarrow}{G}_{\mathrm{EM}}^{(1)}\left(\mathbf{x}-\mathbf{x}^{\prime}, \omega_{1}\right) \stackrel{\leftrightarrow}{G}_{\mathrm{EM}}^{(1)}\left(\mathbf{x}_{1}-\mathbf{x}^{\prime}, \omega_{2}\right),
$$


where

$$
\chi^{(2)}\left(\omega_{1}, \omega_{2}\right)=\int_{0}^{\infty} d \omega_{1}^{\prime} \int_{0}^{\infty} d \omega_{2}^{\prime} v^{(2)}\left(\omega_{1}^{\prime}, \omega_{2}^{\prime}\right) v^{(1)}\left(\omega_{1}^{\prime}\right) v^{(1)}\left(\omega_{2}^{\prime}\right) \frac{1}{\omega_{1}^{\prime 2}-\left(\omega_{1}+i 0^{+}\right)^{2}} \frac{1}{\omega_{2}^{\prime 2}-\left(\omega_{2}+i 0^{+}\right)^{2}}
$$

is the second-order susceptibility. Also, we note that in deriving Eq. (35), only leading terms have been kept and very small terms have been ignored. In order to make contact with standard notation, let us recall the definition of the second-order polarization within the framework of response theory:

$$
\mathbf{P}^{(2)}(t)=\int_{-\infty}^{t} \int_{-\infty}^{t} d t d t^{\prime} \chi^{(2)}\left(t-t^{\prime}, t-t^{\prime \prime}\right) \mathbf{E}\left(t^{\prime}\right) \mathbf{E}\left(t^{\prime \prime}\right)+\mathbf{P}_{N}^{(2)}(t)
$$

where $\mathbf{P}_{N}^{(2)}$ is the noise operator up to the second order of nonlinearity. It is easy to show that Eq. (36) in the time domain can be written as

$$
\chi^{(2)}\left(t_{1}, t_{2}\right)=\int_{0}^{\infty} d \omega_{1} \int_{0}^{\infty} d \omega_{2} v^{(2)}\left(\omega_{1}, \omega_{2}\right) v^{(1)}\left(\omega_{1}\right) v^{(1)}\left(\omega_{2}\right) \frac{\sin \omega_{1} t_{1}}{\omega_{1}} \frac{\sin \omega_{2} t_{2}}{\omega_{2}} \Theta\left(t_{1}\right) \Theta\left(t_{2}\right),
$$

which is consistent with the results that have been reported in [28]. Following this procedure, we find the susceptibility up to the $n$th order as follows:

$$
\chi^{(n)}\left(\omega_{1}, \ldots, \omega_{n}\right)=\int_{0}^{\infty} d \omega_{1}^{\prime} \cdots \int_{0}^{\infty} d \omega_{n}^{\prime} v^{(n)}\left(\omega_{1}^{\prime}, \ldots, \omega_{n}^{\prime}\right) v^{(1)}\left(\omega_{1}^{\prime}\right) \cdots v^{(1)}\left(\omega_{n}^{\prime}\right) \frac{1}{\omega_{1}^{\prime 2}-\left(\omega_{1}+i 0^{+}\right)^{2}} \cdots \frac{1}{\omega_{n}^{\prime 2}-\left(\omega_{n}+i 0^{+}\right)^{2}},
$$

which in the time domain can be expressed as

$$
\chi^{(n)}\left(t_{1}, \ldots, t_{n}\right)=\int_{0}^{\infty} d \omega_{1} \cdots \int_{0}^{\infty} d \omega_{n} v^{(n)}\left(\omega_{1}, \ldots, \omega_{n}\right) v^{(1)}\left(\omega_{1}\right) \cdots v^{(1)}\left(\omega_{n}\right) \frac{\sin \omega_{1} t_{1}}{\omega_{1}} \cdots \frac{\sin \omega_{n} t_{n}}{\omega_{n}} \Theta\left(t_{1}\right) \cdots \Theta\left(t_{n}\right) .
$$

The defined susceptibility contains all optical properties of the medium and vanishes for negative values of $t$ as a consequence of causality. The susceptibility has also the properties of a response function, i.e., $\chi^{(n)}\left(-\omega_{1}^{*}, \ldots,-\omega_{n}^{*}\right)=\chi^{(n) *}\left(\omega_{1}, \ldots, \omega_{n}\right)$, and the Kramers-Kronig relations in a nonlinear medium are satisfied $[42,43]$. Now, if we are given a definite $n$th order susceptibility for the medium, then we can invert the relations [Eq. (40)], and using Eq. (26), the corresponding coupling function $v^{(n)}$ can be found as

$$
v^{(n)}\left(\omega_{1}, \omega_{2}, \ldots, \omega_{n}\right)=\frac{\sqrt{2^{n} \omega_{1} \omega_{2} \cdots \omega_{n}} \operatorname{Im}\left[\chi^{(n)}\left(\omega_{1}, \omega_{2}, \ldots, \omega_{n}\right)\right]}{\left.(\pi)^{n / 2} \sqrt{\operatorname{Im}\left[\chi^{(1)}\left(\omega_{1}\right)\right] \operatorname{Im}\left[\chi^{(1)}\left(\omega_{2}\right)\right] \cdots \operatorname{Im}\left[\chi^{(1)}\left(\omega_{n}\right)\right.}\right]},
$$

where $\operatorname{Im}\left[\chi^{(n)}\right]$ for $n=1$ is exactly the imaginary part of the linear susceptibility, and for $n>2$, we have

$$
\begin{aligned}
\operatorname{Im}\left[\chi^{(n)}\left(\omega_{1}, \ldots, \omega_{n}\right)\right] & \equiv \int_{0}^{\infty} d t_{1} \cdots \int_{0}^{\infty} d t_{n} \chi^{(n)}\left(t_{1}, \ldots, t_{n}\right) \sin \omega_{1} t_{1} \cdots \sin \omega_{n} t_{n} \\
& =\frac{\omega_{1} \omega_{2} \cdots \omega_{n}}{(2 \pi)^{n}} \int_{-\infty}^{\infty} d \omega_{1}^{\prime} \cdots \int_{-\infty}^{\infty} d \omega_{n}^{\prime} \chi^{(n)}\left(\omega_{1}^{\prime}, \ldots, \omega_{n}^{\prime}\right) \frac{1}{\omega_{1}^{2}-\left(\omega_{1}^{\prime}-i 0^{+}\right)^{2}} \cdots \frac{1}{\omega_{n}^{2}-\left(\omega_{n}^{\prime}-i 0^{+}\right)^{2}} .
\end{aligned}
$$

From Eqs. (41) and (42), it is clear that the $n$ th-order coupling function is an odd function in frequencies. Therefore, the $n$ th-order coupling function will be an even (odd) function if $n$ is even (odd). Using this symmetry property, Eq. (34) can be rewritten as

$$
\begin{aligned}
\Delta_{\mathrm{NL}}^{(n)}(\omega)= & \frac{1}{\pi\left(2 \pi^{2}\right)^{n-1}} \int_{-\infty}^{\infty} d \omega_{1} \int_{-\infty}^{\infty} d \omega_{2}^{\prime} \int_{-\infty}^{\infty} d \omega_{2} \cdots \int_{-\infty}^{\infty} d \omega_{n}^{\prime} \int_{-\infty}^{\infty} d \omega_{n} \frac{\left\{\operatorname{Im}\left[\chi^{(n)}\left(\omega_{1}, \omega_{2}, \ldots, \omega_{n}\right)\right]\right\}^{2}}{\operatorname{Im}\left[\chi^{(1)}\left(\omega_{1}\right)\right] \cdots \operatorname{Im}\left[\chi^{(1)}\left(\omega_{n}\right)\right]} \\
& \times \frac{1}{\omega_{1}-\left(\omega-\omega_{2}^{\prime}-\cdots-\omega_{n}^{\prime}+i 0^{+}\right)} \frac{1}{\omega_{2}-\left(\omega_{2}^{\prime}+i 0^{+}\right)} \cdots \frac{1}{\omega_{n}-\left(\omega_{n}^{\prime}+i 0^{+}\right)}
\end{aligned}
$$

In order to illustrate the relations between the coupling functions and the electric susceptibility of the medium, let us restrict our attention to a single resonance electric susceptibility which can be obtained in the extended Lorentz oscillator model. The Lorentz model of the atom, which treats the atom as a harmonic oscillator, is known to provide a very good description of the linear optical properties of atomic vapors and of nonmetallic solids. The linear contribution to the susceptibility is given by

$$
\chi^{(1)}(\omega)=\frac{N\left(e^{2} / m\right)}{D(\omega)},
$$

where $N$ is the number density of atoms, and $D(\omega)$ is defined as

$$
D(\omega)=\omega_{0}^{2}-\omega^{2}-2 i \gamma \omega
$$


where $\omega_{0}$ is the transverse resonance frequency, and $\gamma$ is the absorption parameter. Now, we consider the extended Lorentz model by allowing the possibility of a nonlinearity in the restoring force exerted on the electron. The present model applies for only one resonance frequency, but it cannot properly describe the complete resonance nature of the nonlinear susceptibility, such as the possibility of simultaneous one- and two-photon resonances [44]. For instance, in the case of generation of a mode with added frequencies, the second-order susceptibility can be described as [44]

$$
\chi^{(2)}\left(\omega_{1}, \omega_{2}\right)=\frac{N a\left(e^{3} / m^{2}\right)}{D\left(\omega_{1}\right) D\left(\omega_{2}\right) D\left(\omega_{1}+\omega_{2}\right)},
$$

where $a$ is a parameter that characterizes the strength of the nonlinearity. Now, by substituting Eqs. (44) and (45) into (41), the coupling function $v^{(2)}$ can be obtained as

$$
v^{(2)}\left(\omega_{1}, \omega_{2}\right)=\frac{a e\left[\beta\left(\omega_{0},-\omega_{1}, \omega_{2}, \gamma\right) D\left(\omega_{1}+\omega_{2}\right) D^{*}\left(\omega_{1}+\omega_{2}\right)-\beta\left(\omega_{0}, \omega_{1}, \omega_{2}, \gamma\right) D\left(-\omega_{1}+\omega_{2}\right) D^{*}\left(-\omega_{1}+\omega_{2}\right)\right]}{2 \pi m \gamma \sqrt{D\left(\omega_{1}\right) D^{*}\left(\omega_{1}\right) D\left(\omega_{2}\right) D^{*}\left(\omega_{2}\right)} D\left(\omega_{1}+\omega_{2}\right) D^{*}\left(\omega_{1}+\omega_{2}\right) D\left(-\omega_{1}+\omega_{2}\right) D^{*}\left(-\omega_{1}+\omega_{2}\right)}
$$

where

$$
\beta\left(\omega_{0}, \omega_{1}, \omega_{2}, \gamma\right)=\left(\omega_{0}^{2}-\omega_{1}^{2}\right)\left(\omega_{0}^{2}-\omega_{2}^{2}\right)\left[\omega_{0}^{2}-\left(\omega_{1}+\omega_{2}\right)^{2}\right] \quad-4 \gamma^{2}\left[\omega_{1}\left(\omega_{0}^{2}-\omega_{2}^{2}\right)+\omega_{2}\left(\omega_{0}^{2}-\omega_{1}^{2}\right)\right]\left(\omega_{1}+\omega_{2}\right) .
$$

\section{CAlCulating THE CASimir FORCE}

\section{A. General formalism}

In this section, we calculate the Casimir force for the simplest configuration consisting of two perfectly conducting plates separated by a nonlinear medium of width $h$, as depicted in Fig. 1. For this purpose, we consider the electromagnetic field as having transverse magnetic (TM) and transverse electric (TE) modes. These modes are represented by scalar fields which satisfy the Dirichlet

$$
\varphi\left(X_{\alpha}\right)=0
$$

or Neumann

$$
\partial_{n} \varphi\left(X_{\alpha}\right)=0
$$

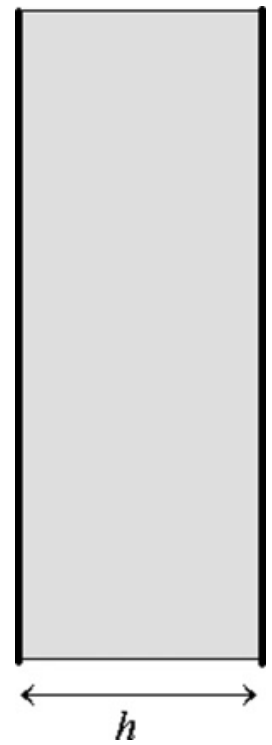

FIG. 1. Nonlinear dielectric slab. boundary conditions, where $X_{\alpha}(\alpha=1,2)$ is an arbitrary point on the conducting plates. To obtain the partition function from the Lagrangian, we apply the Wick rotation $(t \rightarrow i \tau)$ and change the signature of space time from Minkowski's to Euclidean. The Dirichlet or Neumann boundary conditions can be represented by auxiliary fields $\psi_{\alpha}\left(X_{\alpha}\right)$ as [45]

$\delta\left(\varphi\left(X_{\alpha}\right)\right)=\int \mathcal{D}\left[\psi_{\alpha}\left(X_{\alpha}\right)\right] \exp \left(i \int d X_{\alpha} \psi\left(X_{\alpha}\right) \varphi\left(X_{\alpha}\right)\right)$

and

$$
\begin{aligned}
& \delta\left(\partial_{n} \varphi\left(X_{\alpha}\right)\right) \\
& \quad=\int \mathcal{D}\left[\psi_{\alpha}\left(X_{\alpha}\right)\right] \exp \left(i \int d X_{\alpha} \partial_{n} \psi\left(X_{\alpha}\right) \varphi\left(X_{\alpha}\right)\right) .
\end{aligned}
$$

Using Eqs. (51) and (52), the partition functions can be written as

$$
\begin{aligned}
& Z_{D}=Z_{0}^{-1} \int \mathcal{D}[\varphi] \prod_{a=1}^{2} \mathcal{D}\left[\psi_{\alpha}\left(X_{\alpha}\right)\right] e^{S_{D}[\varphi]}, \\
& \left.Z_{N}=Z_{0}^{-1} \int \mathcal{D}[\varphi] \prod_{a=1}^{2} \mathcal{D}\left[\psi_{\alpha}\left(X_{\alpha}\right)\right]\right) e^{S_{N}[\varphi]},
\end{aligned}
$$

where $S_{D}(\varphi)$ and $S_{N}(\varphi)$ are defined respectively by

$$
\begin{aligned}
S_{D}[\varphi]= & \int d^{(n+1)} x\{\mathcal{L}(\varphi(x)) \\
& \left.+\varphi(x) \sum_{\alpha=1}^{2} \int d^{(n)} X \delta\left(X-X_{\alpha}\right) \psi_{\alpha}(x)\right\}
\end{aligned}
$$

and

$$
\begin{aligned}
S_{N}[\varphi]= & \int d^{(n+1)} x\{\mathcal{L}(\varphi(x)) \\
& \left.+\varphi(x) \sum_{\alpha=1}^{2} \int d^{(n)} X \delta\left(X-X_{\alpha}\right) \partial_{n} \psi_{\alpha}(x)\right\} .
\end{aligned}
$$


By comparing the Eqs. (55) and (56) with Eq. (6), we can rewrite Eqs. (55) and (56) as

$$
Z_{D}=\int \prod_{\alpha=1}^{2} \mathcal{D}\left[\psi_{\alpha}(x)\right] Z\left(\sum_{\alpha=1}^{2} \int d^{n} X \delta\left(X-X_{\alpha}\right) \psi_{\alpha}(X)\right)
$$

and

$$
Z_{N}=\int \prod_{\alpha=1}^{2} \mathcal{D}\left[\psi_{\alpha}(x)\right] Z\left(\sum_{\alpha=1}^{2} \int d^{n} X \delta\left(X-X_{\alpha}\right) \partial_{n} \psi_{\alpha}(X)\right),
$$

where $Z\left[\sum_{\alpha=1}^{2} \int d^{n} X_{\alpha} \delta\left(X-X_{\alpha}\right) \psi_{\alpha}(X)\right] \quad$ and $Z\left[\sum_{\alpha=1}^{2}\right.$ $\left.\int d^{n} X_{\alpha} \delta\left(X-X_{\alpha}\right) \partial_{n} \psi_{\alpha}(X)\right]$ are the generating functionals of interacting fields defined in Eq. (18) with imaginary time. From Eqs. (19), (57), and (58), the respective partition functions can be written as

$$
Z_{D}=\int \prod_{\alpha=1}^{2} \mathcal{D}\left[\psi_{\alpha}\left(X_{\alpha}\right)\right] e^{i S_{D}\left(\psi_{1}, \psi_{2}\right)}
$$

and

$$
Z_{N}=\int \prod_{\alpha=1}^{2} \mathcal{D}\left[\psi_{\alpha}\left(X_{\alpha}\right)\right] e^{l S_{N}\left(\psi_{1}, \psi_{2}\right)}
$$

where

$$
\begin{aligned}
& i S_{D}\left(\psi_{1}, \psi_{2}\right)=i J_{D} \mathcal{G} J_{D}, \\
& i S_{N}\left(\psi_{1}, \psi_{2}\right)=i J_{N} \mathcal{G} J_{N},
\end{aligned}
$$

and $J_{D}(X)$ and $J_{N}(X)$ are, respectively, defined by

$$
\begin{gathered}
J_{D}(X)=\int d^{n} X \delta\left(X-X_{\alpha}\right) \psi_{\alpha}(X), \\
J_{N}(X)=\int d^{n} X \delta\left(X-X_{\alpha}\right) \partial_{n} \psi_{\alpha}(X) .
\end{gathered}
$$

We will define the function $\mathcal{G}$ shortly. The partition functions defined by Eqs. (59) and (60) are calculated straightforwardly by [45]

$$
\begin{aligned}
& Z_{D}=\frac{1}{\sqrt{\operatorname{det} \Gamma_{D}(x, y, h)}}, \\
& Z_{N}=\frac{1}{\sqrt{\operatorname{det} \Gamma_{N}(x, y, h)}},
\end{aligned}
$$

where

$$
\begin{gathered}
\Gamma_{D}(x, y, h)=\left[\begin{array}{ll}
\mathcal{G}(x-y, 0) & \mathcal{G}(x-y, h) \\
\mathcal{G}(x-y, h) & \mathcal{G}(x-y, 0)
\end{array}\right], \\
\Gamma_{N}(x, y, h)=\left[\begin{array}{ll}
-\partial_{z}^{2} \mathcal{G}(x-y, 0) & -\partial_{z}^{2} \mathcal{G}(x-y, h) \\
-\partial_{z}^{2} \mathcal{G}(x-y, h) & -\partial_{z}^{2} \mathcal{G}(x-y, 0)
\end{array}\right],
\end{gathered}
$$

and $\mathcal{G}$ is the Green's function of the fields after a Wick rotation. Now, in order to calculate the Casimir force, we define the effective action as

$$
S_{\text {eff }}=-\ln Z_{D}[h],
$$

from which the Casimir force can be obtained easily as

$$
F=\frac{\partial S_{\text {eff }}(h)}{\partial h} .
$$

It is easy to show that the Dirichelet and Neumann boundary conditions are formally the same and, when the plates are complete conductors, lead to the same result. For the sake of brevity, in what follows, we only calculate the Casimir force for Dirichelet boundary conditions; its generalization to Neumann boundary conditions is straightforward.

\section{B. Linear and nonlinear effects of the medium $(T=0)$}

To calculate the Casimir energy in presence of a nonlinear medium boundary, conditions are imposed on the electromagnetic field, and the $\Gamma$ tensor can be written as

$$
\Gamma_{\mathrm{EM}, \mathrm{EM}}(x, y, h)=\left[\begin{array}{ll}
\mathcal{G}_{\mathrm{EM}, \mathrm{EM}}(x-y, 0) & \mathcal{G}_{\mathrm{EM}, \mathrm{EM}}(x-y, h) \\
\mathcal{G}_{\mathrm{EM}, \mathrm{EM}}(x-y, h) & \mathcal{G}_{\mathrm{EM}, \mathrm{EM}}(x-y, 0)
\end{array}\right] .
$$

Now, to obtain the Casimir force, we proceed in Fourier space since the $\Gamma$ tensor is diagonal in this space. The Fourier transformation of $\mathcal{G}_{\mathrm{EM}, \mathrm{EM}}(x-y, h)$ is

$$
\begin{aligned}
\mathcal{G}_{\mathrm{EM}, \mathrm{EM}}(p, q, h) & =\int d x \int d y e^{i p x+i q y} G_{\mathrm{EM}, \mathrm{EM}}(x-y, h) \\
& =\frac{\exp \left\{-h \sqrt{\left[\epsilon\left(i p_{0}\right)+\Delta_{\mathrm{NL}}^{(2)}\left(i p_{0}\right)+\cdots+\Delta_{\mathrm{NL}}^{(n)}\left(i p_{0}\right)\right] p_{0}^{2}+p_{1}^{2}+p_{2}^{2}}\right\}}{2 \sqrt{\left[\epsilon\left(i p_{0}\right)+\Delta_{\mathrm{NL}}^{(2)}\left(i p_{0}\right)+\cdots+\Delta_{\mathrm{NL}}^{(n)}\left(i p_{0}\right)\right] p_{0}^{2}+p_{1}^{2}+p_{2}^{2}}}(2 \pi)^{3} \delta(p+q),
\end{aligned}
$$

where $p=\left(p_{0}, \mathbf{p}\right)$ in which $\mathbf{p}$ is a vector parallel to the conductors, and $p_{0}$ is the temporal component of $p$. Thus, in this case, the Casimir force is

$$
F_{c}=-\int \frac{d^{3} p}{(2 \pi)^{3}}\left[\frac{\mathcal{Q}(p)}{e^{2 \mathcal{Q}(p) h}-1}\right],
$$

where

$$
\mathcal{Q}(p)=\sqrt{\mathbf{p}^{2}+p_{0}^{2}\left[\epsilon\left(i p_{0}\right)+\Delta_{\mathrm{NL}}^{(2)}\left(i p_{0}\right)+\cdots+\Delta_{\mathrm{NL}}^{(n)}\left(i p_{0}\right)\right]} .
$$


It is easy to show that the Casimir force in the presence of a nonlinear medium [Eq. (73)] is similar to the Casimir force in the presence of a linear one; the only difference is in the definition of $\mathcal{Q}(p)$. In the absence of the nonlinearity, i.e., $\Delta_{\mathrm{NL}}^{(2)}(\omega)=\cdots=\Delta_{\mathrm{NL}}^{(n)}(\omega)=0$, the original Casimir force between two plates in the presence of a linear medium is recovered.

In order to illustrate the effects of the nonlinearity of the medium on the Casimir force, again we restrict our attention to the second-order susceptibility. To simplify the problem, instead of using the complex susceptibility [Eq. (46)], we consider the following susceptibilities in the time domain:

$$
\chi^{(1)}\left(t_{1}\right)=\alpha \sin \Omega t_{1} \Theta\left(t_{1}\right),
$$

$$
\chi^{(2)}\left(t_{1}, t_{2}\right)=\beta \sin \Omega t_{1} \sin \Omega t_{2} \Theta\left(t_{1}\right) \Theta\left(t_{2}\right),
$$

where $\alpha$ and $\beta$ are constants. Therefore, by using Eqs. (42) and (43), the first-order correction to the Casimir force due to the second-order nonlinearity of the medium can be expressed as

$$
\Delta_{\mathrm{NL}}^{(2)}(\omega)=\frac{2 i \beta^{2} \Omega^{2}}{\alpha \omega\left(4 \Omega^{2}-\omega^{2}\right)} .
$$

Now, substituting the above equation into Eq. (73), the Casimir force in the presence of second-order nonlinearity can be written as

$$
F_{c}=-\int \frac{d^{3} p}{(2 \pi)^{3}}\left\{\frac{\sqrt{\mathbf{p}^{2}+p_{0}^{2}\left[1+\frac{\alpha \Omega}{\left(\Omega^{2}+p_{0}^{2}\right)}+\frac{2 \beta^{2} \Omega^{2}}{\alpha p_{0}\left(4 \Omega^{2}+p_{0}^{2}\right)}\right]}}{\exp \left\{2 h \sqrt{\mathbf{p}^{2}+p_{0}^{2}\left[1+\frac{\alpha \Omega}{\left(\Omega^{2}+p_{0}^{2}\right)}+\frac{2 \beta^{2} \Omega^{2}}{\alpha p_{0}\left(4 \Omega^{2}+p_{0}^{2}\right)}\right]}-1\right.}\right\} .
$$

\section{Finite temperature}

Our considerations so far have been restricted to zero temperature. In fact, the temperature corrections to the Casimir force turned out to be negligible in experiments $[9,46,47]$ where the measurements were performed in the separation range $h<1 \mathrm{~mm}$. But, at $h>1 \mathrm{~mm}$, as in [8], the temperature corrections make larger contributions to the zero-temperature force between perfect conductors. The generalization of the formalism to this case is straightforward. The inclusion of temperature may be done in the usual manner [3,48-50]. The finite temperature expression can be found by replacing the frequency integral by a sum over Matsubara frequencies according to the rule

$$
\hbar \int_{0}^{\infty} \frac{d \xi}{2 \pi} f(\iota \xi) \rightarrow k_{B} T \sum_{l=0}^{\infty^{\prime}} f\left(\iota \xi_{l}\right), \quad \xi_{l}=2 \pi k_{B} T l / \hbar
$$

where $T$ and $k_{B}$ are the temperature and Boltzmann constant, respectively, and the prime over the summation means the zeroth term should be given half weight as is conventional. Therefore, the Casimir force at finite temperature can be expressed as

$$
F_{c}=-\frac{k_{B} T}{4 \pi^{2} \hbar} \sum_{l=0}^{\infty^{\prime}} \int d^{2} \mathbf{p}\left[\frac{\mathcal{Q}(p)}{e^{2 \mathcal{Q}(p) h}-1}\right] .
$$

\section{CONCLUSION}

Based on a canonical approach and using path-integral techniques, the electromagnetic field in a nonlinear dielectric is quantized, and the Casimir force, in the presence of a nonlinear medium at finite temperature, is calculated.
[1] H. B. G. Casimir, Proc. K. Ned. Akad. Wet. 51, 793 (1948).

[2] E. M. Lifshitz, Zh. Eksp. Teor. Fiz. 29, 94 (1956) [Sov. Phys. JETP 2, 73 (1956)]; I. D. Dzyaloshinskii, E. M. Lifshitz, and L. P. Pitaevskii, Usp. Fiz. Nauk 73, 381 (1961) [Sov. Phys. Usp. 4, 153 (1961)].

[3] K. A. Milton, L. L. Deraad, and J. Schwinger, Ann. Phys. (NY) 115, 388 (1978).

[4] M. S. Tomaš, Phys. Rev. A 66, 052103 (2002); Phys. Lett. A 342, 381 (2005).

[5] C. Raabe, L. Knoll, and D.-G. Welsch, Phys. Rev. A 68, 033810 (2003); C. Raabe and D.-G. Welsch, ibid. 71, 013814 (2005).

[6] M. J. Spaarnay, Physica 24, 751 (1958).

[7] E. S. Sabisky and C. H. Anderson, Phys. Rev. A 7, 790 (1973).

[8] S. K. Lamoreaux, Phys. Rev. Lett. 78, 5 (1997); 81, 5475 (1998).
[9] U. Mohideen and A. Roy, Phys. Rev. Lett. 81, 4549 (1998); A. Roy and U. Mohideen, ibid. 82, 4380 (1999).

[10] K. A. Milton, The Casimir Effect: Physical Manifestation of Zero-Point Energy (World Scientific, Singapore, 2001).

[11] S. C. Lim and L. P. Teo, New J. Phys. 11, 013055 (2009).

[12] P. W. Milonni, The Quantum Vacuum (Academic, San Diego, 1994).

[13] M. Krech, The Casimir Effect in Critical Systems (World Scientific, Singapore, 1994).

[14] V. M. Mostepanenko and N. N. Trunov, The Casimir Effect and its Applications (Clarendon Press, Oxford, 1997).

[15] H. B. Chan, V. A. Aksyuk, R. N. Kleiman, D. J. Bishop, and F. Capasso, Science 291, 1941 (2001); Phys. Rev. Lett. 87, 211801 (2001).

[16] K. Busch et al., Phys. Rep. 444, 101 (2007). 
[17] J. D. Joannopoulos, R. D. Meade, and J. N. Winn, Photonic Crystals: Molding the Flow of Light (Princeton University Press, Princeton, NJ, 1995).

[18] K. Sakoda, Optical Properties of Photonic Crystals (SpringerVerlag, Berlin, Heidelberg, New York, 2001).

[19] M. Hillery and L. D. Mlodinow, Phys. Rev. A 30, 1860 (1984).

[20] P. D. Drummond, Phys. Rev. A 42, 6845 (1990).

[21] I. Abram and E. Cohen, Phys. Rev. A 44, 500 (1991).

[22] L. M. Duan and G. C. Guo, Phys. Rev. A 56, 925 (1997).

[23] E. Schmidt et al., J. Mod. Opt. 45, 377 (1998).

[24] E. Schmidt, J. Jeffers, S. M. Barnett, L. Knöll, and D.-G. Welsch, J. Mod. Opt. 45, 377 (1998).

[25] S. Scheel and D.-G. Welsch, J. Phys. B 39, S711 (2006).

[26] S. Scheel and D.-G. Welsch, Phys. Rev. Lett. 96, 073601 (2006).

[27] S. Scheel and S. Y. Buhmann, Acta Phys. Slov. 58(5), 675 (2008).

[28] M. Amooshahi and E. Amooghorban, Ann. Phys. (NY) 325, 1976 (2010).

[29] H. Li and M. Kardar, Phys. Rev. Lett. 67, 3275 (1991); Phys. Rev. A 46, 6490 (1992).

[30] R. Golestanian and M. Kardar, Phys. Rev. A 58, 1713 (1998).

[31] T. Emig, A. Hanke, R. Golestanian, and M. Kardar, Phys. Rev. Lett. 87, 260402 (2001).

[32] T. Emig, A. Hanke, R. Golestanian, and M. Kardar, Phys. Rev. A 67, 022114 (2003).

[33] F. Kheirandish, M. Soltani, and J. Sarabadani, Phys. Rev. A 81, 052110 (2010).
[34] B. Huttner and S. M. Barnett, Phys. Rev. A 46, 4306 (1992); Europhys. Lett. 18, 487 (1992).

[35] L. G. Suttorp and M. Wubs, Phys. Rev. A 70, 013816 (2004).

[36] F. Kheirandish and M. Amooshahi, Phys. Rev. A 74, 042102 (2006).

[37] F. Kheirandish and M. Soltani, Phys. Rev. A 78, 012102 (2008).

[38] L. Ryder, Quantum Field Theory, 2nd ed. (Cambridge University Press, Cambridge, 1996).

[39] R. Matloob and H. Falinejad, Phys. Rev A 64, 042102 (2001).

[40] C. Raabe and D.-G. Welsch, Phys. Rev. A 71, 013814 (2005).

[41] S. Jiang and P. Gallion, J. Opt. Soc. Am. B 26, 902 (2009).

[42] K.-E. Peiponen, Phys. Rev. B 37, 6463 (1988); K.-E. Peiponen, E. M. Vartiainen, and T. Asakura, Opt. Rev. 4, 433 (1997).

[43] F. Bassani and S. Scandolo, Phys. Rev. B 44, 8446 (1991).

[44] R. W. Boyd, Nonlinear Optics, 2nd ed. (Academic, New York, 2003).

[45] J. Sarabadani and M. F. Miri, Phys. Rev. A 74, 023801 (2006).

[46] A. Roy, C.-Y. Lin, and U. Mohideen, Phys. Rev. D 60, 111101(R) (1999).

[47] B. W. Harris, F. Chen, and U. Mohideen, Phys. Rev. A 62, 052109 (2000).

[48] G. D. Mahan, Many Particle Physics, 3rd ed. (Plenum, New York, 2000).

[49] E. M. Lifshitz and L. P. Pitaevskii, Statistical Physics (Pergamon Press, Oxford, 1980), Part 2.

[50] J. I. Kapusta, Finite-Temperature Field Theory (Cambridge University Press, Cambridge, 1989). 